\title{
Binary-Coded Monitoring of a Behavioral Sequence by Cells in the Pre-Supplementary Motor Area
}

\author{
Keisetsu Shima ${ }^{1}$ and Jun Tanji ${ }^{1,2}$ \\ ${ }^{1}$ Department of Physiology, Tohoku University School of Medicine, Sendai 980-8575, Japan and ${ }^{2}$ Brain Science Research Center, Tamagawa University \\ Research Institute, Tokyo 194-8610, Japan
}

\begin{abstract}
To regulate the temporal structure of a series of behavioral sequences involving multiple actions, it is essential to monitor the progress of the entire behavioral process. To identify the involvement of three cortical motor areas in monitoring behavioral sequences, we examined neuronal activity while monkeys sequentially performed a series of motor tasks in accordance with a predetermined behavioral schedule that included a numerical structure. We found that neurons in the pre-supplementary motor area exhibited activity that appeared to monitor the performance of the behavioral trials in a binary-coded manner. One-half of the activity represented odd-numbered trials within a behavioral sequence, whereas the other one-half represented even-numbered trials. Such neuronal activity, resembling the operation of binary counting elements widely used for constructing artificial computing devices, was rare in the supplementary motor area or in the primary motor cortex.
\end{abstract}

Key words: cerebral cortex; monkey; binary coding; neuronal activity; pre-supplementary motor area; behavioral sequence

\section{Introduction}

The ability to carry out sequences of actions is fundamental to most normal primate behaviors (Lashley, 1951). The temporal organization of actions required to achieve sequential behaviors requires the involvement of neural activity at cortical (Tanji, 2001) and subcortical (Braitenberg et al., 1997; Beiser and Houk, 1998) levels. Previous studies have found neural elements that code temporal sequences of actions (Barone and Joseph, 1989; Mushiake et al., 1990; Kermadi and Joseph, 1995; Grafton et al., 1998; Shima and Tanji, 2000). Neuronal activity signaling the beginning (Shima et al., 1996) and end (Fujii and Graybiel, 2003) of a behavioral sequence has also been reported, in line with theoretical models of sequential behavior that exploit explicit designations of the start and end states (Dehaene and Changeux, 1997). If an additional requirement is posed to temporally arrange a series of behavioral trials, each requiring a sequence of movements, over an extended time period, then subjects must keep track of the entire series of trials to build a temporal pattern of successive trials. In such a case, the CNS is required to monitor the complete progress of each series of trials from beginning to end. How do cortical neurons achieve the numerical monitoring of a behavioral sequence of that sort? To answer this question, we examined neuronal activity in three frontal cortical areas of monkeys while they performed a sequential motor task following a predetermined trial schedule. We show that cells in the pre-

Received Sept. 30, 2005; revised Jan. 21, 2006; accepted Jan. 22, 2006.

This work was supported by Grant-in-Aid for Scientific Research on Priority Areas "Integrative Brain Research" from the Ministry of Education, Culture, Sports, Science and Technology of Japan (Grant 17021004). We thank M. Kurama and Y. Takahashi for technical assistance.

Correspondence should be addressed to Jun Tanji, Tamagawa University Research Institute, Brain Research Center, 6-1-1 Tamagawa Gakuen, Machida, Tokyo 194-8610, Japan. E-mail: tanji@lab.tamagawa.ac.jp.

DOI:10.1523/JNEUROSCI.4161-05.2006

Copyright $\odot 2006$ Society for Neuroscience $\quad$ 0270-6474/06/262579-04\$15.00/0 supplementary motor area (pre-SMA) in particular appeared to monitor behavioral sequences in a binary mode, with some cells representing odd-numbered trials within the behavioral sequence and others representing even-numbered trials.

\section{Materials and Methods}

Behavioral procedures. We used two monkeys (Macaca fuscata), cared for in accordance with the National Institutes of Health Guide for the Care and Use of Laboratory Animals, to perform two different motor tasks described below. We trained the monkeys to perform three movements (push, pull, or turn a handle) with the right arm in six different orders. While sitting in a primate chair, each monkey was required to place the handle in a neutral position and wait 2.5-4.5 s (hold period) for the first movement-triggering signal (a high-pitched tone). When the animal performed the first movement, a mechanical device returned the handle to the neutral position. While keeping the handle in this position, the animal had to wait $1-1.4 \mathrm{~s}$ for the second movement-triggering signal, and then another 1-1.4 s for the third. A series of three correct movements was rewarded $500 \mathrm{~ms}$ later with the delivery of applesauce. The intertrial interval was $2-4.5 \mathrm{~s}$. Initially, the correct movements were indicated with green (for turn), red (for push), and yellow (for pull) lights. The lights came on individually at the time of each movement, together with the movement-triggering tone signal. During this period of five visually guided trials, the animal had to learn the correct sequence, after which the sequential motor task was performed from memory. In the memory-guided trials, only the tone signal was given as the movement trigger, with no lights. After completing six trials of the memorized sequential task, randomly flashing lights (for $2 \mathrm{~s}$ ) signaled the end of the current sequence and the beginning of the next sequence. After the lights flashed, the wait period (2.5-4.5 s) for the subsequent trial began. Consequently, a particular sequence of movements was performed in blocks. Each block consisted of 11 trials with a particular sequence of the three movements, 5 trials under visual guidance and 6 with no visual cues, followed by the next block with a different sequence of movements. The order of appearance of different sequences (e.g., turn-push-pull within a block) varied unpredictably in a pseudorandom order. At least two 
blocks of trials of each sequence of movements were included in a data file while recording from individual cells.

Surgery, acquisition of neuronal data, and histology. After completing the behavioral training, an acrylic recording chamber $(40 \times 30$ $\mathrm{mm}$ ) and head fixation bolts were implanted on the skull under aseptic conditions. The monkeys were anesthetized with pentobarbital sodium $(30 \mathrm{mg} / \mathrm{kg}$, i.m.), after induction with ketamine hydrochloride $(8 \mathrm{mg} / \mathrm{kg}$, i.m.) with atropine sulfate. Antibiotics and analgesics were used to prevent postsurgical infection and pain. Standard electrophysiological techniques for single-cell recording were used to record from the left pre-SMA, SMA, and primary motor cortex (MI) (for the definitions of the SMA and pre-SMA, see Matsuzaka et al., 1992). After complete recovery from surgery, neuronal activity was recorded in the medial part of the frontal cortex using glass-insulated Elgiloy microelectrodes inserted through the dura. After collecting the neuronal data, the recording sites were verified histologically by examining Nisslstained brain sections.

Data analysis. Our database included neurons from which activity was recorded during more than two blocks of trials for each of six different sequences under either visual or memory guidance. We defined three periods for data analysis: the control period (500 ms during the initial hold period), the preparatory period ( $2 \mathrm{~s}$ before the first GO signal), and the motor response period (from the start of the GO signal until completion of the third movement). Neuronal activity was defined as taskrelated if the discharge rate during either the preparatory or motor response periods differed significantly from that recorded during the control period (Wilcoxon signed-ranks test; $\alpha=0.05)$. The sampling of neurons depended entirely on this test for task relevance. Subsequently, to statistically assess how behavioral parameters were related to the activity of cortical neurons, we performed a multiple linear regression analysis using the following regression model (Zar, 1999): Firing rate $=\beta_{0}+\beta_{1} \times$ (ordinal trial numbers $)+\beta_{2} \times$ (task condition) $+\beta_{3}$ (movement sequence).

The firing rate was calculated in individual trials during either the preparatory or motor response period. In this model of equation, $\beta_{0}$ is the intercept and $\beta_{1}, \beta_{2}$, and $\beta_{3}$ are regression coefficients. The first categorical factor was the ordinal position of the trial in a sequence (with levels usually corresponding to the 1st through 11th trials). The second categorical factor was the behavioral condition with two levels (visually or memory guided). The third factor was the sequence of movements (push, pull, or turn) with six levels, corresponding to individual sequences. We calculated the probability ( $p$ ) that each coefficient equaled zero. If $p<0.01$, the neuronal activity was accepted as reflecting each factor. If, for instance, the probability ( $p$ value) that the coefficient $\beta_{1}$ equaled zero was $<0.01$, we judged that the firing rate reflected the ordinal trial number. In this report, we focused on neuronal activity that was considered to reflect the ordinal trial number. For these trial-number selective neurons, as the next step of analysis, we compared activity during the performance of trials categorized as odd-numbered trials in a block with activity during even-numbered trials. For this comparison, we used the Mann-Whitney $U$ test ( $p<0.05$ for significant differences), using the data obtained during either the preparatory or motor-response period. Subsequently, as a quantitative measure of odd-even number selectivity, we calculated an odd/even selectivity index as follows: Selectivity index $=\left|\left(\mathrm{FR}_{\text {odd }}-\mathrm{FR}_{\text {even }}\right)\right| /\left(\mathrm{FR}_{\text {odd }}+\mathrm{FR}_{\text {even }}\right)$.

In this equation, $\mathrm{FR}_{\text {odd }}$ and $\mathrm{FR}_{\text {even }}$ signify the firing rates during the odd- and even-numbered trials. We also examined whether neuronal
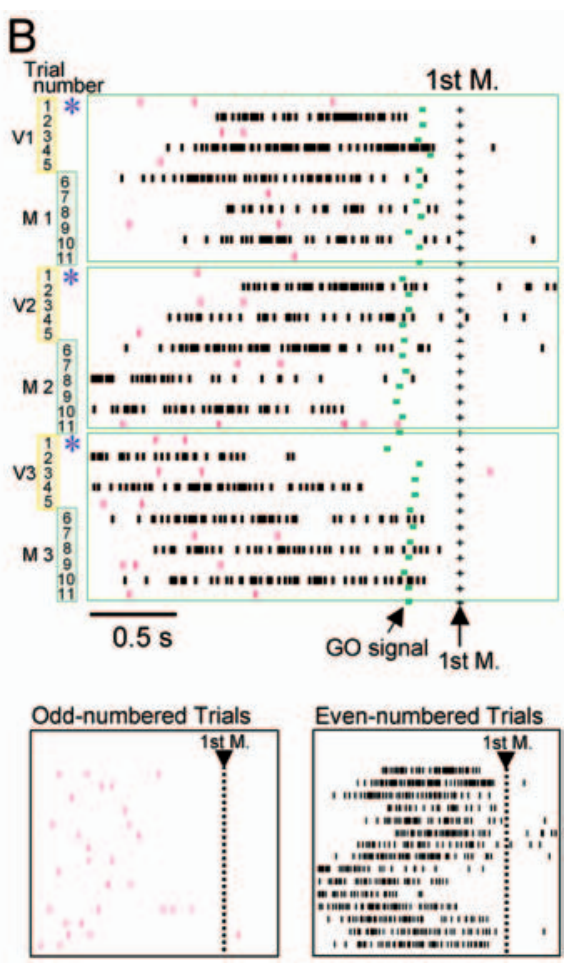

1 st M.

ii

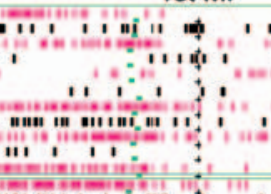

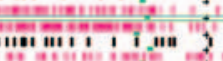

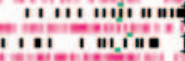

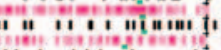

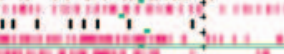

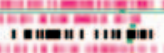

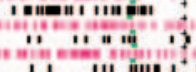

, ilime

$\because \because 1, y=$
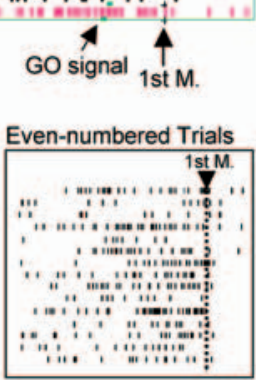

cell exhibits a high level of discharges in odd-numbered trials, but a low level in even-numbered trials, and is always reset at the first trial when a new activity in even-numbered trials. During recording of this cell, the sequences are push-pull-turn during V1/M1, push-turn-pull ush during V3/M3.

activity was selective for a particular ordinal number of trials (1st through 11 th). We did not find activity that was selective for trial numbers ranging from 2 nd to 10 th, although we found selectivity appearing after the completion of the 11th trial and before the execution of the 1st trial (as reported by Shima et al., 1996). Electromyographic recordings were made from muscles in the digits, wrist, elbow, shoulder, trunk, and paravertebral area, and the data were digitized and analyzed quantitatively in a similar manner to the neuronal data. We did not find any muscle activity that was selective for particular trial numbers. Furthermore, we did not find any behavioral differences between the two monkeys (patterns of muscle activity or reaction times of individual motor tasks under visual or memory guidance). Both monkeys failed to show preferences for handedness.

\section{Results}

We recorded cellular activity from the pre-SMA of the two monkeys under this behavioral condition and found 401 task-related cells ( $\alpha=0.05$; Wilcoxon signed-ranks test). We found that $23 \%$ of the task-related cells in this area $(n=70$ or $31 \%$ for monkey A; $n=21$ or $12 \%$ for monkey B) exhibited two distinct levels of activity alternately during the performance of successive trials. In these cells, trial-by-trial analysis revealed the alternation of highand low-level discharges in successive trials $(p<0.05$; MannWhitney $U$ test): high-level activity followed by low-level activity or vice versa, as seen in Figure 1. In the majority of cases (93\%), the level of alternating activity was always reset at the start of a new trial sequence, regardless of the sequence to be performed. Therefore, in one-half of such cells (52\%), activity was greater in 

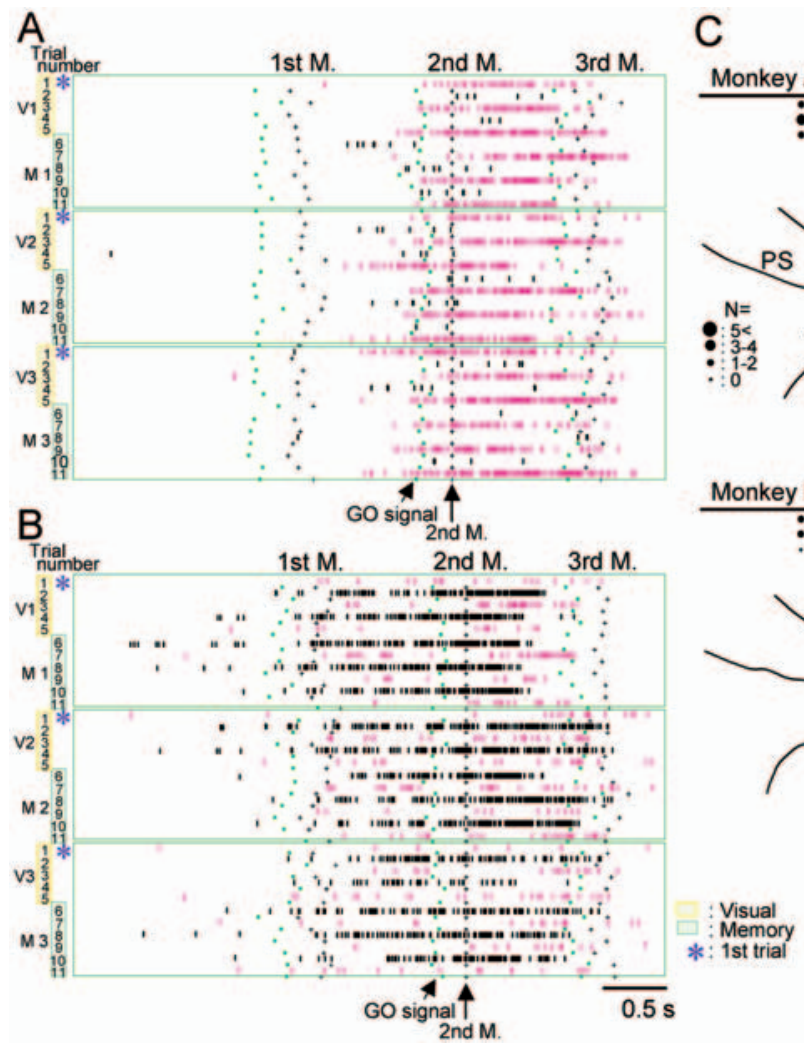

Figure 2. Discharges of two pre-SMA cells exhibiting selectivity for odd- $(\boldsymbol{A})$ and even-numbered $(\boldsymbol{B})$ trials during execution of sequential movements. The order of required hand movements during each V/M block corresponds to a different sequence pattern. The display formats are the same as in Figure 1, except that discharges are aligned at the onset of the second of the three movements. C, Cortical surface maps showing recording sites, corresponding to the location of cells that exhibited selectivity for odd/even number of trials. The size of the filled circle is proportional to the number of selective cells at each penetration site. The arrows in each map indicate a boundary of the SMA and pre-SMA. PS, Principal sulcus; ARC, arcuate sulcus; CS, central sulcus.
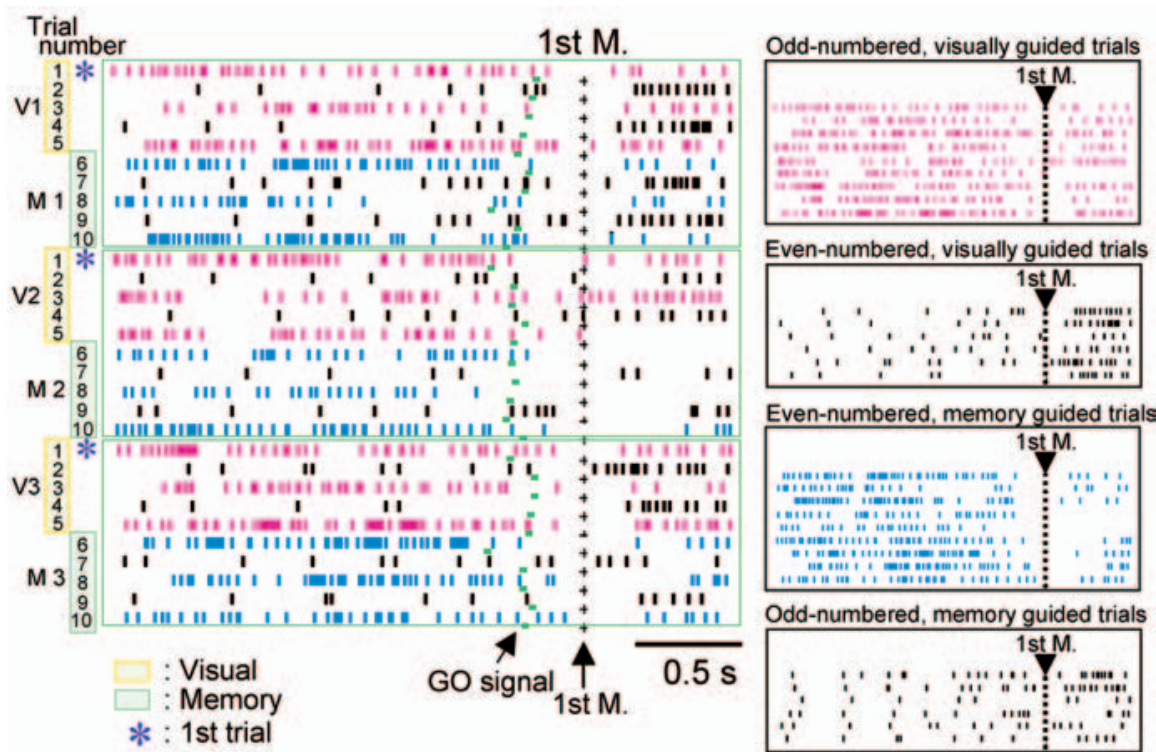

Figure 3. Discharges of a pre-SMA cell whose activity is reset at the transition from visual guidance to memory guidance of a motor sequence. The activity is also reset at the transition of sequences.

odd-numbered trials (as in Fig. 1A), whereas in the other onehalf, activity was greater in even-numbered trials (Fig. $1 B$ ). Such binary trial-selective activity was observed in either the preparatory period preceding the occurrence of the first movement in the sequence (58\%; as shown in Fig. 1), during execution of the three movements $(24 \%$; as presented in Fig. 2), or during both $(18 \%)$.

It is important to note that the odd/even number selectivity was observed despite the fact that each block of visually guided (V)/ memory-based (M) trial corresponds to a different sequence pattern the monkey had to perform. We also found that the selectivity remained unchanged across several different sequence blocks (including 12-24 V/M blocks). Interestingly, the binary-coded activity pattern deteriorated in trials when the monkey made an error, or stopped attending to the task. For a limited number of cells $(n=$ 12 ), we increased the number of either visually guided or memory-based trials (up to 10 trials each). The binary-selective nature of activity remained despite these alterations. Recording sites of neurons with binary selectivity are shown in Figure 2C. In both monkeys, the binary-selective cells were scattered throughout the pre-SMA without apparent clustering, although the proportion of selective cells among the total of task-related cells was greater in monkey $\mathrm{A}\left(p<0.01 ; \chi^{2}\right.$ test $)$.

In the majority of binary-modulated cells, the activity level was reset only at the transition of motor sequences, as described above. In eight neurons, however, the level of activity was also reset at the transition of behavioral conditions when the guidance of the motor sequence changed from visually signaled to memory-based. As a result, neuronal activity appeared to be greater (or lower) for the first, third, and fifth trials than for the others, exclusively under either visual or memory guidance. An example of such activity is shown in Figure 3.

For the majority of binary-selective cells in the pre-SMA, the selectivity index was $>0.6$, whereas for the majority of SMA cells, the selectivity index was $<0.6$ (Fig. $4 A$ ). We also examined neuronal activity in the SMA and MI, and identified 503 and 288 neurons, respectively, that were taskrelated. We rarely found binary-selective neurons in the SMA $(n=15 ; 4 \%)$ or MI $(n=1 ;<1 \%)$, as shown in Figure $4 B$.

\section{Discussion}

In this study, we found that the activity of a sizeable number of neurons in the preSMA was modulated in a manner such that it alternated during the successive performance of trials, and was reset when a new movement sequence started. Because we failed to detect any trial-selective alterations in muscle activity or animal's motor behavior (monitored with a video system), any trivial explanations for the modulation resulting from the motor habits of the animal are unlikely. Therefore, we propose that the modulation 
A
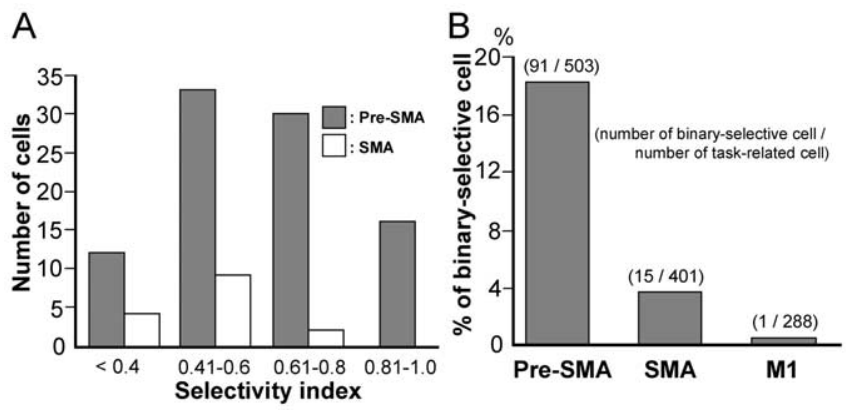

Figure 4. Distribution of binary selectivity index and binary-selective cells in three motor areas. $\boldsymbol{A}$, The number of binary-selective cells in the pre-SMA (shaded bars) and SMA (open bars). $\boldsymbol{B}$, Percentages of binary-selective cells in the pre-SMA, SMA, and M1.

reflects monitoring of the behavioral sequences in a binary-coded manner, thereby providing a basis for generating a signal to terminate the current behavioral routine and to initialize the next routine, thus updating relevant information (motor sequence in the present behavioral task).

Although the pre-SMA is defined as a cortical motor area, previous studies have reported that the pre-SMA is less involved in movement execution than the SMA (Matsuzaka et al., 1992; Tanji, 1996). Rather, the pre-SMA has been implicated in such cognitive aspects of motor behaviors as motor planning, task switching (Matsuzaka and Tanji, 1996; Rushworth et al., 2002), procedural learning (Nakamura et al., 1998; Sakai et al., 1999), the retrieval of sensory signals (Hoshi and Tanji, 2004), temporal-order coding of multiple movements (Shima and Tanji, 2000), the organization of action sequences (Kennerley et al., 2004), and intention for action (Lau et al., 2004). In this study, we found a new aspect of the involvement of the pre-SMA in behavioral control. What is the function of neuronal activity characterized as binary-coded monitoring of behavioral sequences? One possibility is that the activity serves to provide numerical information necessary to keep a register of the progress of events in each segment of the trials. Such information seems necessary to construct a structure in a behavioral segment, providing a basis for signaling behavioral epochs. A possible use of neural elements involved in the numerical processing of individual trials may be to generate signals designating the start and end of a series of behavioral sequences across behavioral trials, which is vital for sequential behavior (Dehaene and Changeux, 1997; Fujii and Graybiel, 2003). We hypothesize that the binary-coded monitoring of successive trials serves to provide the numerical information required, accumulating information about the number of trials accomplished. Such numericalprocessing elements in a cortical area may be relevant to both "start" and "end" signals in a sequence of behavioral trials (Shima et al., 1996; Shima and Tanji, 2000). Under our behavioral conditions, we did not find the representation of numbers themselves, as reported previously (Dehaene et al., 1998; Nieder et al., 2002; Sawamura et al., 2002; Eger et al., 2003; Nieder and Miller, 2003). The absence of digital-number representations seems to arise from the fact that behavioral counting itself was not a requirement to achieve the behavioral task in our study.

Binary-coding elements are widely used for artificial counting devices or computers. Therefore, it is interesting to find neural elements operating in a binary-coded manner in the brain. This finding invites studies of human subjects to search for similar binary-coding neural elements and determine their possible functions. It would also be of interest to explore neuronal circuits, such as corticobasal ganglia loops, that produce a two-state alternating level of cellular activity (Houk and Wise, 1995).

\section{References}

Barone P, Joseph JP (1989) Prefrontal cortex and spatial sequencing in macaque monkey. Exp Brain Res 78:447-464.

Beiser DG, Houk JC (1998) Model of cortical-basal ganglionic processing: encoding the serial order of sensory events. J Neurophysiol 79:3168-3188.

Braitenberg V, Heck D, Sultan F (1997) The detection and generation of sequences as a key to cerebellar function: experiments and theory. Behav Brain Sci 20:229-245.

Dehaene S, Changeux JP (1997) A hierarchical neuronal network for planning behavior. Proc Natl Acad Sci USA 94:13293-13298.

Dehaene S, Dehaene-Lambertz G, Cohen L (1998) Abstract representations of numbers in the animal and human brain. Trends Neurosci 21:355-361.

Eger E, Sterzer P, Russ MO, Giraud AL, Kleinschmidt A (2003) A supramodal number representation in human intraparietal cortex. Neuron 37:719-725.

Fujii N, Graybiel AM (2003) Representation of action sequence boundaries by macaque prefrontal cortical neurons. Science 301:1246-1249.

Grafton ST, Hazeltine E, Ivry RB (1998) Abstract and effector-specific representations of motor sequences identified with PET. J Neurosci 18:9420-9428.

Hoshi E, Tanji J (2004) Area-selective neuronal activity in the dorsolateral prefrontal cortex for information retrieval and action planning. J Neurophysiol 91:2707-2722.

Houk JC, Wise SP (1995) Distributed modular architectures linking basal ganglia, cerebellum, and cerebral cortex: their role in planning and controlling action. Cereb Cortex 5:95-110.

Kennerley SW, Sakai K, Rushworth MFS (2004) Organization of action sequences and the role of the pre-SMA. J Neurophysiol 91:978-993.

Kermadi I, Joseph JP (1995) Activity in the caudate nucleus of monkey during spatial sequencing. J Neurophysiol 74:911-933.

Lashley KS (1951) The problem of serial order in behavior. In: Cerebral mechanisms in behavior (Jeffress LA, ed), pp 112-136. New York: Wiley.

Lau HC, Rogers RD, Haggard P, Passingham RE (2004) Attention to intention. Science 303:1208-1210.

Matsuzaka Y, Tanji J (1996) Changing directions of forthcoming arm movements: neuronal activity in the presupplementary and supplementary motor area of monkey cerebral cortex. J Neurophysiol 76:2327-2342.

Matsuzaka Y, Aizawa H, Tanji J (1992) A motor area rostral to the supplementary motor area (presupplementary motor area) in the monkey: neuronal activity during a learned motor task. J Neurophysiol 68:653-662.

Mushiake H, Inase M, Tanji J (1990) Selective coding of motor sequence in the supplementary motor area of the monkey cerebral cortex. Exp Brain Res 82:208-211.

Nakamura K, Sakai K, Hikosaka O (1998) Neuronal activity in medial frontal cortex during learning of sequential procedures. J Neurophysiol 80:2671-2687.

Nieder A, Miller EK (2003) Coding of cognitive magnitude: compressed scaling of numerical information in the primate prefrontal cortex. Neuron 37:149-157.

Nieder A, Freedman DJ, Miller EK (2002) Representation of the quantity of visual items in the primate prefrontal cortex. Science 297:1709-1711.

Rushworth MF, Hadland KA, Paus T, Sipila PK (2002) Role of the human medial frontal cortex in task switching: a combined fMRI and TMS study. J Neurophysiol 87:2577-2592.

Sakai K, Hikosaka O, Miyauchi S, Sasaki Y, Fujimaki N, Putz B (1999) Presupplementary motor area activation during sequence learning reflects visuo-motor association. J Neurosci 19:RC1(1-6).

Sawamura H, Shima K, Tanji J (2002) Numerical representation for action in the parietal cortex of the monkey. Nature 415:918-922.

Shima K, Tanji J (2000) Neuronal activity in the supplementary and presupplementary motor areas for temporal organization of multiple movements. J Neurophysiol 84:2148-2160.

Shima K, Mushiake H, Saito N, Tanji J (1996) Role for cells in the presupplementary motor areas in updating motor plans. Proc Natl Acad Sci USA 93:8694-8698.

Tanji J (1996) New concepts of the supplementary motor area. Curr Opin Neurobiol 6:782-787.

Tanji J (2001) Sequential organization of multiple movements: involvement of cortical motor areas. Annu Rev Neurosci 24:631-651.

Zar JH (1999) Biostatistical analysis. Upper Saddle River, NJ: Prentice Hall. 